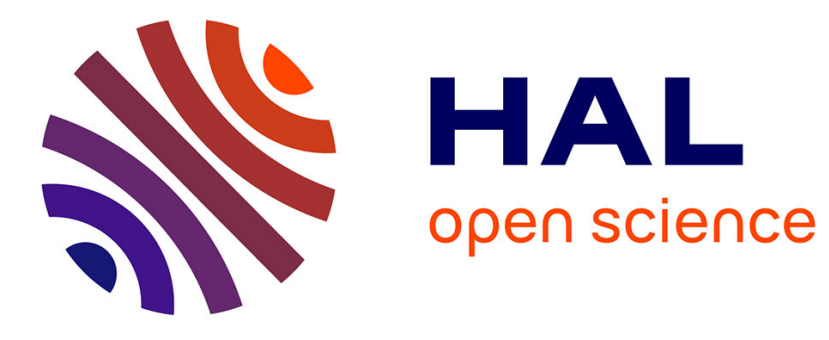

\title{
États-Unis. Le mariage religieux des couples de même sexe
}

\author{
Baptiste Coulmont
}

\section{To cite this version:}

Baptiste Coulmont. États-Unis. Le mariage religieux des couples de même sexe. Virginie Descoutures, Marie Digoix, Éric Fassin et Wilfried Rault. Mariages et homosexualités dans le monde. L'arrangement des normes familiales, Autrement, pp.73-82, 2007. halshs-00599986

\section{HAL Id: halshs-00599986 \\ https://shs.hal.science/halshs-00599986}

Submitted on 21 Dec 2012

HAL is a multi-disciplinary open access archive for the deposit and dissemination of scientific research documents, whether they are published or not. The documents may come from teaching and research institutions in France or abroad, or from public or private research centers.
L'archive ouverte pluridisciplinaire HAL, est destinée au dépôt et à la diffusion de documents scientifiques de niveau recherche, publiés ou non, émanant des établissements d'enseignement et de recherche français ou étrangers, des laboratoires publics ou privés. 


\section{États-Unis : le mariage religieux des couples de même sexe \\ Baptiste Coulmont}

Communication au colloque : « Normes et mariage », École normale supérieure, INED, Paris.

Version préliminaire d'un texte publié sous les références suivantes :

«États-Unis. Le mariage religieux des couples de même sexe », in Virginie Descoutures, Marie Digoix, Éric Fassin et Wilfried Rault (dirs.), Mariages et homosexualités dans le monde. L'arrangement des normes familiales, Paris, Éditions Autrement, 2008, p.73-82

Pour les institutions religieuses aux Etats-Unis, l'ouverture du mariage aux couples de même sexe est à première vue une question externe, dans la mesure où une grande partie des débats porte sur le mariage civil. Mais c'est aussi une question éminemment interne. Il s'agit de la place, dans la société, du discours religieux et des normes édictées par les Églises (quand ces dernières considèrent, par exemple, l'homosexualité comme un péché). De la place des mécanismes de régulation des hérésies (quand des prêtres ou des pasteurs, gais ou non, s'autorisent à célébrer des cérémonies interdites). De la place d'identités ou de subjectivités contemporaines - séculières, mondaines et modernes - dans les discours religieux. Question interne, enfin, parce que ces interrogations se posent au sein d'un champ religieux, où les prises de positions prennent sens les unes relativement aux autres.

\section{Une question externe?}

L'ouverture du mariage aux couples du même sexe touche directement les Eglises. Aux États-Unis, un mariage civil peut en effet être célébré par un membre du clergé agissant en tant qu' "agent de l'État». Toute reformulation, par le droit séculier, des caractéristiques des candidats au mariage civil aura donc une influence - directe ou indirecte - sur les candidatures à une célébration religieuse. La sous-traitance religieuse de la célébration du mariage civil fait alors de ces cérémonies des objets religieux ambigus. Le mariage fait partie du menu de services qu'une institution religieuse offre habituellement à ses fidèles. Mais ce n'est pas principalement sur ce point qu'insistent les Eglises : tout d'abord c'est un élément standardisé sur lequel il n'est pas évident d'être compétitif; ensuite, il tient souvent plus de l'acte administratif que de la profession de foi.

L'internalisation progressive de la question du mariage se perçoit bien au cours des quinze dernières années. À la suite d'une décision de la cour suprême de Hawaï, en 1993, Baehr v. Lewin, le mariage des couples de même sexe est devenu un enjeu juridique sur lequel se sont penchés juristes, législateurs et sociologues. À mesure que la possibilité du mariage (civil) des couples de même sexe se précisait, le débat prenait également une dimension religieuse. Depuis le milieu des années 1990, les différentes Églises chrétiennes, de même que la plupart des courants du judaïsme américain, ont ainsi été amenés à prendre position face à l'essor manifeste des cérémonies (religieuses) d'union, un essor lui-même produit par les mobilisations intervenues dans le champ séculier.

Ainsi, outre quelques interventions des autorités religieuses, on voit l'émergence de questions très pratiques: si le mariage civil est ouvert aux couples du même sexe et qu'arrivent des demandes de célébrations de ce genre, que faire ?, s'interroge l'évêque épiscopalien de Hawaï au milieu des années 1990. L'embarras suscité par cette situation est à 
double titre révélateur : d'un côté, il souligne la différence théorique entre mariage religieux et civil, de l'autre il montre la précarité de ces frontières, dans les usages religieux.

Question apparemment externe, le mariage des couples du même sexe devient alors rapidement une question «interne ». L'Église méthodiste unie vote ainsi en 1996 l'interdiction pour ses pasteurs de célébrer des « unions homosexuelles », une décision alors en phase avec le vote la même année, par le Congrès américain, de la "Loi de Défense du Mariage », autorisant les Etats à ne pas reconnaître des mariages du même sexe s'ils venaient à être légalisés dans d'autres Etats.

Au cours des dix dernières années, l'irruption des unions civiles dans l'éventail des régimes conjugaux légaux a accentué la proximité des débats séculiers et religieux. Celles qui ont été instituées dans le Vermont en 1999 donnent aux couples de même sexe les droits que le mariage attribue aux couples de sexe différent (ce qui, bien sûr, ne comprend pas les droits liés au niveau fédéral). Aujourd'hui, à l'instar du Vermont, le New Jersey, le Connecticut et le New Hampshire ont mis en place ces unions civiles, et le Massachusetts a ouvert en 2004 le mariage aux couples de même sexe. Les personnes dûment ordonnées par leur Église peuvent les célébrer « au nom de l'État». Du point de vue de l'État, religion et couples homosexuels ne forment pas une antithèse.

Les Revised Statutes du New Jersey précisent ainsi que «n'importe quel ministre de n'importe quelle religion est par la présente autorisé à solenniser mariage ou union civile entre personnes pouvant en droit entrer dans le mariage ou l'union civile; toute société, institution ou organisation religieuse dans cet État peut unir par les liens du mariage ou de l'union civile de telles personnes, en accord avec les règles et les coutumes de cette société, institution ou organisation ${ }^{1}$.»

Les membres du clergé deviennent ainsi des célébrants possibles d'unions homosexuelles reconnues par l'État. Certains pasteurs, peu nombreux, ont alors demandé que leur Église sorte totalement du dispositif de sous-traitance - pour les couples de sexes différents tout comme pour les couples de même sexe. Le but était ici de rendre au mariage célébré à l'église son caractère religieux. Mais d'autres ont, au contraire, embrassé la nouvelle possibilité qu'offrait l'État. Dans celui du Vermont, la création des unions civiles fut très conflictuelle, mais depuis lors, on a depuis assisté à la rapide routinisation de ces cérémonies. Les logiques de mobilisation, d'opposition, de conflit, qui reposaient sur des formes «prophétiques» insistant soit sur l'absolue nécessité de l'ouverture du mariage, soit sur les risques pour la santé sociale ou le salut individuel, bref, sur le caractère extraordinaire de cette innovation socio-légale, ont rapidement laissé la place à des logiques «bureaucratiques» ou «traditionnalisées »: les unions civiles font désormais partie du paysage, y compris du paysage religieux pour certaines Églises locales (épiscopaliennes, congrégationnalistes, unitariennes...).

L' « union civile», catégorie d'État, devient insensiblement une catégorie du discours religieux, mais d'un discours forgé par la pratique.

\section{Le rôle des identités}

Le mariage n'est pas qu'un statut impliquant l'État. C'est aussi un moyen de rendre publiques les relations amoureuses. Les assemblées locales de fidèles ont donc eu à gérer la

\footnotetext{
1 «[E]very minister of every religion, are hereby authorized to solemnize marriage or civil union between such persons as may lawfully enter into the matrimonial relation or civil union; and every religious society, institution or organization in this State may join together in marriage or civil union such persons according to the rules and customs of the society, institution or organization » (New Jersey Revised Statutes, 17. R.S.37:1-13).
} 
publicité des relations amoureuses homosexuelles, avant même que ne soit entreprise toute action en justice de la part de couples privés du mariage civil.

Il existe une préhistoire du mariage religieux des couples du même sexe, une préhistoire qui montre que les cérémonies religieuses d'unions homosexuelles s'analysent également comme l'un des modes de gestion privilégiés de l'homosexualité par certaines Églises. Des acteurs religieux, tenus d'innover en matière d'action pastorale et d'élaboration théologique afin de transformer l'homosexualité en forme " bénigne » de variation sexuelle, ont pu s'appuyer sur le mariage - ou les bénédictions d'union - pour diffuser un message. Dans cette perspective pragmatique, l'accès des gais et des lesbiennes au mariage - et plus spécifiquement au mariage religieux - a pu apparaître comme un enjeu d'importance.

Bénédictions d'unions de couples de même sexe et mobilisations d'opposition à ces unions ont contribué à l'entrée d'identités sexuelles modernes dans les discours religieux. En effet, les débats n'ont pas été structurés autour des anciennes catégories théologiques et juridiques de "sodomie " ou de " fornication », mais autour des revendications politiques de groupes homosexuels. Dans les années 1970 ont lieu les premières expérimentations religieuses autour des cérémonies d'union et les premiers célébrants tentent d'acclimater leurs rites et leur théologie à des identités publiques construites hors des Églises, par exemple en intégrant pleinement les termes "homosexuels » et « hétérosexuels» dans leur réflexion. L'identité gaie qui se redéfinit alors repose sur le «coming out» individuel, sur l'existence d'un mouvement collectif de libération, mais aussi d'associations de gais et de lesbiennes aux buts variés. Identité sociale transversale, elle s'accommode d'une facette religieuse : foi et homosexualité ne sont pas forcément incompatibles

S'établissent, à partir du début des années 1970, certains compromis locaux, où les mariages religieux ne sauraient avoir de valeur civile pour les couples de même sexe, et où l'on ne parle d'ailleurs pas vraiment de "mariage ». Les termes retenus (" union sainte», «cérémonie d'union», «bénédiction d'amour», «alliance sacrée »...) inscrivent la cérémonie dans «autre chose» que le mariage. On pourrait aussi repérer les mêmes négociations contraintes entre les pasteurs et leur " hiérarchie » : pendant longtemps, il s'agit d'éviter de parler de mariage, cependant qu'une pratique se développe au fil des années 1970 et 1980, sous le regard de celles et ceux qui s'y opposeront plus tard, mais aussi en dessous du champ d'action de leur " radar ». Les oppositions au mariage religieux des couples de même sexe, quand elles se manifestent, se font sur le mode du dégoût : le mariage gai rebute, mais il n'est pas perçu comme remettant en cause l'institution ou les principes fondateurs de la civilisation. Les «radars » de la droite conservatrice n'y décèlent pas encore une question sociale. Jusqu'au début des années 1990, certains pasteurs (qui deviendront parfois évêques) vont pouvoir célébrer religieusement l'union de deux hommes ou deux femmes. Dans certaines Eglises, après plus de trente-cinq ans de célébrations, ces unions gaies ou lesbiennes ne font pas seulement partie du paysage, elles participent de l'identité profonde de ces Eglises.

Une facette spécifique aux débats religieux (qui ne se retrouve pas dans les débats séculiers) est toutefois présente : celle de la sexualité des célébrants, hommes ou femmes... et plus largement de l'homosexualité du clergé. Si le mariage gai est une question institutionnelle, celui des simples fidèles est, somme toute, accessoire. En revanche, puisque les prêtres, les pasteurs et les rabbins ne doivent pas avoir de relations sexuelles hors mariage, si l'on ordonne des prêtres gais, il faut alors les marier - à l'exception bien sûr de ceux de l'Eglise catholique. A l'inverse, le fait que l'on ne marie pas les couples de même sexe montre bien qu'il ne faut pas ordonner de pasteurs gais, soulignent les mouvements conservateurs au sein d'Églises plutôt libérales. Au cours des années 1970, à mesure qu'une bonne partie des Églises protestantes avalise l'entrée des femmes dans les fonctions de 
pasteur, les anciennes tensions liées au sexe des clercs font place à de vives discussions autour de leur sexualité.

La création d'un rite du mariage homosexuel permettrait de dissoudre le cercle vicieux qui relie la question de l'accès au pastorat des homosexuels et celle de leur sexualité.

Suite à la polémique concernant l'ordination d'une pasteure lesbienne, en 1977, la chambre des évêques épiscopaliens affirme que l'Église « est en droit de réserver sa bénédiction nuptiale au mariage hétérosexuel exclusivement» et que l'ordination des « homosexuels revendicatifs ou pratiquants » (advocating and/or practicing homosexuals) est « inadmissible » : l'ordination et le mariage sont liés, dès l'origine des controverses. Dès lors, à chaque ordination d'un prêtre homosexuel vivant en couple, se pose la question du statut de son union. S'il ne s'agit pas d'un mariage, alors, les relations sexuelles sont interdites. En revanche, si son union était à l'image d'un mariage - une image particulière que cherchent à promouvoir ses défenseurs, fondée sur l'exclusivité des relations sexuelles - ses relations sexuelles seraient avalisées. La question de l'ordination rejoint donc fortement celle du mariage.

Des documents conservés dans les archives de liturgistes épiscopaliens souhaitant créer un rituel d'union mettent en lumière le contexte des réflexions cléricales sur les unions de même sexe et soulignent encore le lien entre ordination et mariage. Une partie des expérimentations rituelles a été en effet réalisée pour la célébration de l'union de pasteurs homosexuels.

Il faut alors toujours garder à l'esprit que la question du mariage est tout autant une question "pastorale» (qui concerne les fidèles et leur traitement par le clergé) qu'une question "cléricale" (qui concerne les prêtres et leurs époux/épouses). Et c'est cette question « interne » qui tend à mobiliser les prêtres.

Mais à un autre niveau d'analyse, le mariage agit comme le révélateur de la présence d'identités contemporaines, modernes, séculières (et politiques) au sein des Eglises. Ce sont ces identités, peut-être plus que certaines pratiques sexuelles, qui sont violemment refusées, en partie parce qu'elles sont des signes de la sécularisation des catégories d'entendement - y compris au sein des espaces sociaux cléricaux : là aussi, l'homosexuel comme catégorie prend la place du sodomite. Les identités gaies et lesbiennes vont s'opposer et se combiner à d'autres identités... Tout un ensemble de chrétiens « gais » mais conservateurs, de chrétiens souhaitant «sortir» de l'homosexualité, de personnes se revendiquant " ex-gays », essaient de proposer un autre «kit identitaire ». Mais ce travail identitaire conservateur, de manière paradoxale, va renforcer l'inscription de l'homosexualité au cœur des Églises : loin de revenir aux catégories traditionnelles de la théologie, le christianisme conservateur utilise les descriptions sécularisées des identités sexuelles.

Il semblait encore possible, il y a quelques années, de décrire la rencontre de deux mondes opposés et distincts: l'homosexualité d'un côté, la religion de l'autre. Certes, l'homosexualité (le terme lui-même est forgé vers 1870) se constitue socialement au moment même où le processus de sécularisation et de laïcisation s'accélère; mais au cours du $\mathrm{XX}^{\mathrm{e}}$ siècle, alors que la médecine (ainsi que la psychiatrie, la psychologie, les psychothérapies...) mais aussi le droit, cessent de considérer qu'elle entre dans leur champ de compétence, il se passe exactement l'inverse dans le champ religieux. Ce n'est pas seulement parce que l'opposition à l'homosexualité trouve une base religieuse institutionnelle, mais aussi parce qu'elle fait débat, à l'intérieur des Églises, et que ces dernières, surtout parmi des mouvements conservateurs, tentent une construction identitaire alternative. 
Homosexualité identitaire et religion institutionnelle entretiennent donc des relations tendues ${ }^{2}$, conflictuelles, mais qui ne devraient pas cacher ou recouvrir la perméabilité de frontières sociales. Les mouvements «ex-gays», qui proposent des psychothérapies d'inspiration religieuse, prennent comme base d'action une identité publique (ou du moins sa possibilité) : le but ultime est de transformer des homosexuel hommes (peu souvent des femmes) en hétérosexuels, mais en pratique ils contribuent à inscrire ces hommes dans des réseaux - amicaux, religieux, professionnels - où leur identité d' « ex-gay » est centrale. Les Églises « libérales » essaient d'insérer l'homosexualité comme variation « bénigne » d'une orientation sexuelle majoritaire. Et c'est pour ces raisons que l'homosexualité, loin d'être quelque chose d'extérieur qui ne s'attaquerait qu'aux marges des institutions, peut véritablement être utilisée comme une «loupe grossissante» permettant de saisir de l'intérieur le fonctionnement de ces institutions religieuses.

\section{Réguler des hérésies}

Des compromis qui avaient pu paraître acceptables dans un contexte où l'homosexualité était d'abord conçue comme un péché individuel ont cessé de l'être, aux yeux des mouvements conservateurs, dès lors qu'elle est apparue comme « un péché doté d'un mouvement social $»^{3}$.

L'homosexualité à laquelle se sont confrontées l'Église catholique et les «denominations » protestantes américaines n'est plus ce qu'elles considéraient comme un "vice privé », elle est devenue une identité publique.

Dans les Églises au fonctionnement « démocratique »- où un vote majoritaire lors d'une assemblée générale décide de l'évolution doctrinale - la " mise en norme », la création de normes, peut donner lieu à des combats politiques publics. Les assemblées générales d'une partie des Églises américaines proclamèrent alors le caractère hétérosexuel du mariage : l'évidence n'allait plus de soi et devait être écrite noir sur blanc, toute violation par un membre de l'Église de cette norme désormais posée comme telle exposant le contrevenant à une peine religieuse.

Dans les années 1990 vont ainsi se multiplier les usages d'une forme qui avait presque disparu du monde protestant américain, le procès ecclésiastique. L'impact du mariage gai sur les Églises, c'est donc aussi une sorte de judiciarisation. Les interdictions formelles de célébration, à la suite de controverses publiques, ont été suivies d'exemples de désobéissance ecclésiastique de la part de certains pasteurs, qui ont en conséquence été jugés par des cours de justice religieuses. Le mariage des couples de même sexe, pour une partie des Églises n'a donc pas seulement suscité des controverses et des débats publics, mais aussi des procès religieux, qui matérialisaient à un moment donné des modes de gestion de la dissidence.

Le choix de la judiciarisation des conflits n'est pas seulement suivi par des pasteurs "libéraux », souhaitant rendre publique par le scandale une action qu'ils estiment prophétique, au double sens d'inspirée par Dieu et d'avant-garde. Les menaces de schismes, venues du côté conservateur, sont toujours présentes. Dans des Églises parcourues de courants divers et institutionnalisés (par des associations, des magazines, des grandes figures...), le schisme - ou sa menace - est une option possible. Historiquement, elle a souvent été suivie : au moment de la Guerre de Sécession, autour des débats entre modernistes et fondamentalistes, ou de ceux sur l'ordination des femmes... On ne compte donc plus les départs de pasteurs (parfois pour des dénominations plus conservatrices d'un point de vue

2 Pour un exemple, voir l'ouvrage récent de Philip Jenkins, The New Anti-Catholicism : the Last Acceptable Prejudice, Oxford (GB) : Oxford University Press, 2003, qui consacre un chapitre aux relations tendues entre l'Église catholique américaine et certaines associations gaies.

3 Didi Herman, The Anti-Gay Agenda: Orthodox Vision and the Christian Right, Chicago (IL), The University of Chicago Press, 1997, p. 50. 
doctrinal), les sécessions d'assemblées locales, et, plus récemment, les menaces schismatiques de certains diocèses (épiscopaliens) ou de sections régionales.

\section{Conclusions}

J'ai essayé de montrer ici certaines des raisons sociologiques pour lesquelles le mariage des couples de même sexe a été l'occasion de débats religieux, mais aussi de mobilisations collectives aux États-Unis. C'est une question éminemment interne, qui renvoie non seulement aux modes de gestions de l'innovation rituelle, de l'hérésie, mais aussi à la place des identités contemporaines, séculières, dans l'espace religieux.

Parce que le mariage est, aux États-Unis, un objet mixte, séculier en droit, souvent religieux dans sa célébration, il peut être pris comme analyseur de l'état contemporain de la sécularisation, ce processus qui a longtemps constitué la base des réflexions sociologiques sur la religion. À un premier niveau, les oppositions vivaces à l'ouverture du mariage, oppositions souvent religieuses, invoquant un ordre transcendant l'accord démocratique, pourraient faire croire à une dé-sécularisation: elles sont efficaces, suscitent d'importantes mobilisations, structurent certains discours conservateurs. Mais c'est aussi la puissance des catégories séculières de compréhension du monde que ces débats religieux autour du mariage gai révèlent quand ils mobilisent « unions civiles », " homosexualité » ou droit séculier, soit une « sécularisation interne » ${ }^{4}$ des discours religieux.

\footnotetext{
4 Francois-André Isambert, «La sécularisation interne du christianisme », Revue Française de
} Sociologie, 1976, Vol. 17, $\mathrm{n}^{\circ}$ 4, pp. 573-589 


\section{Encadré : des Églises bien différentes}

Il est presque possible de classer différentes Églises chrétiennes en fonction de leurs refus, plus ou moins fréquent, des célébrants homosexuel-le-s ou des unions de même sexe. Des plus libéraux aux plus conservateurs, voici une petite liste (avec la manière de nommer en anglais le clergé) :

Unitariens-universalistes (minister) : accordent l'ordination aux gays ou aux lesbiennes et célèbrent des unions de même sexe. Se situent aux marges du christianisme.

Congrégationnalistes (pastor) : accordent une grande autonomie aux assemblées locales, mais souvent dans un sens "libéral ». Ordinations d'homosexuels et cérémonies d'union sans grand conflit aujourd'hui.

Episcopaliens (priest) : branche américaine de la confession anglicane. Plusieurs évêques (en fonction ou retraités) ont déclaré leur homosexualité. Grande variété des politiques d'ordination ou de célébration, suivant les diocèses. Certains ont des liturgies quasiofficielles.

Presbytériens (pastor) : Conflits profonds autour des questions homosexuelles.

Méthodistes (pastor): Interdiction de l'ordination de pasteurs homosexuels (hommes ou femmes) : du type « don't ask, don't tell » (ne rien demander, ne rien dire : c'est le compromis adopté sous Bill Clinton sur les gays et lesbiennes dans l'armée américaine). Conflits publics au sujet des unions religieuses (plusieurs pasteurs ont été jugés par des tribunaux ecclésiastiques).

Baptistes du Sud (pastor): Opposition frontale à l'homosexualité (certaines assemblées locales ont été exclues (disfellowshipped) pour avoir été jugées trop favorables aux causes gaies).

Catholiques romains (priest): Politique centrale hostile aux manifestations publiques de l'homosexualité.

Une variété aussi importante se retrouve au sein du judaïsme américain, entre rabbins de tendances «reform» ou «conservative » (aux politiques plutôt libérales, malgré le nom) et «orthodox». 